\title{
Multi-physics evaluation of carbonate-rich source rocks from high-resolution images
}

Shannon L. Eichmann (D) and Mita Sengupta, Aramco Americas: Aramco Research Center - Houston, 16300 Park Row Drive, Houston, TX 77084, USA Abdelrahman Kotb, Aramco Americas: Aramco Research Center - Houston, 16300 Park Row Drive, Houston, TX 77084, USA; 609 Richardson Petroleum Engineering Building, 3116 TAMU, 245 Spence Street, College Station, TX 77843, USA

Address all correspondence to Shannon L. Eichmann at shannon.eichmann@aramcoamericas.com

(Received 13 May 2021; accepted 11 0ctober 2021; published online: 9 November 2021)

\section{Abstract}

In unconventional reservoirs, the pore space is hosted by a heterogeneous matrix with various minerals and organic components. This heterogeneity complicates petrophysical interpretation during hydrocarbon exploration. A digital rock physics study of thermal and electrical conductivity was conducted using high-resolution focused ion beam scanning electron microscopy images of carbonate-rich source rocks. Finite-volume simulation results are discussed in context of the sample heterogeneity and anisotropy and supported by comparisons to empirical equations and effective medium theory. The results show how the presence of organic matter, pyrite, and pore constrictions impacts application of empirical equations and simplified models to unconventional reservoirs.

\section{Introduction}

Source rocks are composed of a mixture of inorganic minerals and organic matter that create a composite rock structure with composition, texture, and pore sizes that vary at the nanoand microscales. ${ }^{[1,2]}$ Chemical heterogeneity arises from the changing composition of the inorganic mineral matrix which may include calcite, dolomite, quartz, and clay minerals, in addition to various organic components (i.e., kerogen, bitumen, and pyrobitumen) that are the source of hydrocarbons. ${ }^{[1,3,4]}$ Carbonate-rich source rocks consist of a calcite framework that contains varying amounts of organic components and pores. The pores of reservoir rocks store and transport fluids and those found in source rocks can be contained within the inorganic (i.e., inorganic porosity) or organic phases (i.e., organic porosity) where the organic porosity is developed as the organic matter is converted to hydrocarbons.

Characterizing reservoir and rock properties is a key step when assessing reservoir quality, estimating resources in place, and planning for field development. Determining porosity and permeability are essential to reservoir characterization because these properties affect reserves estimates and hydrocarbon production. However, accurately measuring the permeability of ultra-low permeability rocks, such as source rocks, is challenging. In addition, thermal and electrical conductivity measurements are also of interest, and each can be measured on bulk samples in the lab or measured in the field by logging tools or estimated from petrophysical logs. ${ }^{[5-8]}$ Nevertheless, few techniques exist, which can measure thermal and electrical conductivity at the micro- and nanoscale, the length scales of the source rock heterogeneity. Moreover, bulk measurements do not make it possible to study how the local rock structure and compositional heterogeneity impact these properties.
Scanning Electron Microscopy (SEM) is commonly used for source rock characterization. ${ }^{\left[{ }^{-12]}\right.}$ High-resolution twodimensional (2D) images of the complex, nanometer-scale, heterogeneity of source rocks are taken at the length scale of the heterogeneity of the rock fabric and pore space. These high-resolution images provide important context to evaluate the potential of unconventional reservoirs. Focused Ion Beam SEM (FIB-SEM) imaging provides a means to move beyond 2D imaging to provide three-dimensional (3D) image volumes to capture the rock complexity at high resolution. Image processing methods are then applied to segment the various image components into separate label fields for quantitative analyses. Thus, the amount of the various components (i.e., matrix, organics, and high-density components), porosity, and pore size distribution can be measured and compared with other data at the same scale or at a larger scale.

Digital rock physics (DRP) simulations utilize images to predict the physical properties of rocks. Thus, DRP can complement challenging laboratory measurements or provide estimated properties when accurate laboratory measurements are not available. ${ }^{[13-19]}$ Labeled images from image segmentation are assigned physical properties of the known materials for advanced mathematical simulations of physical characteristics such as flow behavior, electrical and thermal conductivity, and elasticity. ${ }^{[13-17,19]}$ The results of these simulations can then be reviewed in the context of the changing structural framework. A correlative simulation-based study of structural effects on mechanical, thermal, and electrical conductivity, and flow properties has been reported for nanoporous metals. ${ }^{[19]}$ While DRP permeability prediction has been demonstrated for unconventional reservoir rocks, the use of DRP to study the thermal and electrical conductivity is less reported in the literature. ${ }^{[10,20-23]}$ 
In addition, most DRP studies of unconventional reservoir rocks focus on shales or tight carbonates which typically contain limited organic matter (i.e., $<10 \%$ ) and inorganic porosity. This is different from carbonate-rich source rocks which can contain upwards of $15 \%$ organic matter and mainly organic porosity. These structural and compositional differences limit application of prior literature to the study of nano- and microscale behavior of carbonate-rich source rocks which are the focus this work.

This paper demonstrates the use of 3D FIB-SEM imaging and DRP to quantify thermal and electrical conductivity of carbonate-rich source rocks with varying composition and structure. Quantitative image processing provides insight into the compositional and porosity variations and measures the total versus connected (i.e., effective) porosity. Each voxel of the segmented images was assigned thermal and electrical conductivity of the bulk materials, and finite-volume simulations were performed on the full and sub-sectioned rock volumes to provide effective properties in all three directions. The effect of the changing organic content and porosity on the DRP-predicted composite properties is discussed, and the composite properties are compared with empirical correlations and effective medium theory calculations. For carbonate-rich source rocks with pores filled with high salinity water, the thermal conductivity correlates with organic matter content while the electrical conductivity is controlled by the fluid-filled connected porosity rather than the total porosity.

\section{Methods \\ Samples}

Four carbonate-rich source rock samples from the Middle East were selected for this study. The details of the geographic location, geology, and geochemistry can be reviewed in prior literature. ${ }^{[24]}$ The four samples were selected from the same well covering a depth range of less than $250 \mathrm{ft}$.

\section{Scanning electron microscopy and image processing}

Carbonate-rich source rock samples from the Middle East were sub-sectioned and prepared for Focused Ion Beam Scanning Electron Microscopy (FIB-SEM) (Zeiss Crossbeam $540)$. For each sample (H1-H4), a series of 2D image slices were collected using a low accelerating voltage $(0.85-1 \mathrm{kV})$ to reduce charging. Both secondary electron (SE) and backscatter electron (ESB) images were collected and the individual SE and ESB slices are aligned, cropped, filtered, and combined to create a $3 \mathrm{D}$ gray-scale volume (Avizo ${ }^{\circledR}$ and PerGeos ${ }^{\circledR}$, Thermo Fisher Scientific). Each 3D volume $(\sim 1000 \times 1000 \times 1000$ pixels, $10 \mathrm{~nm} /$ pixel, see Table $\mathrm{S} 1$ for specific volume dimensions) was segmented using grayscale intensity ranges to separate four major components: pores, organics, high-density minerals (i.e., pyrite), and matrix minerals (i.e., calcite). To increase sampling, each full $3 \mathrm{D}$ volume was then sub-sectioned into eight equally sized sub-volumes (Fig. 1(b)). The volume fraction of each component in each full image volume and sub-volume was measured (Fig. 2(a)). The pores were separated from each segmented image volume and filtered for corner connectivity (i.e., neighborhood $=26$ ) to measure the total and connected porosity (Figs. 1(c), 2(b)). While not the topic of this paper, accurate alignment and component segmentation are a highly important step to this process. Figure S1 shows example SE, $\mathrm{ESB}$, and segmented images from a few slices from $\mathrm{H} 1$ and $\mathrm{H} 4$ to demonstrate the segmentation results. All data presented are obtained from the segmented volumes including

a

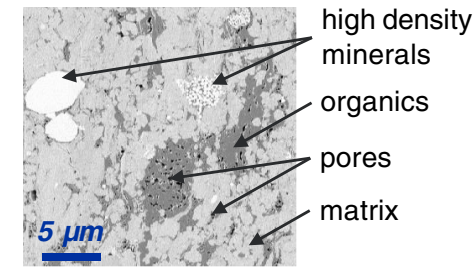

b
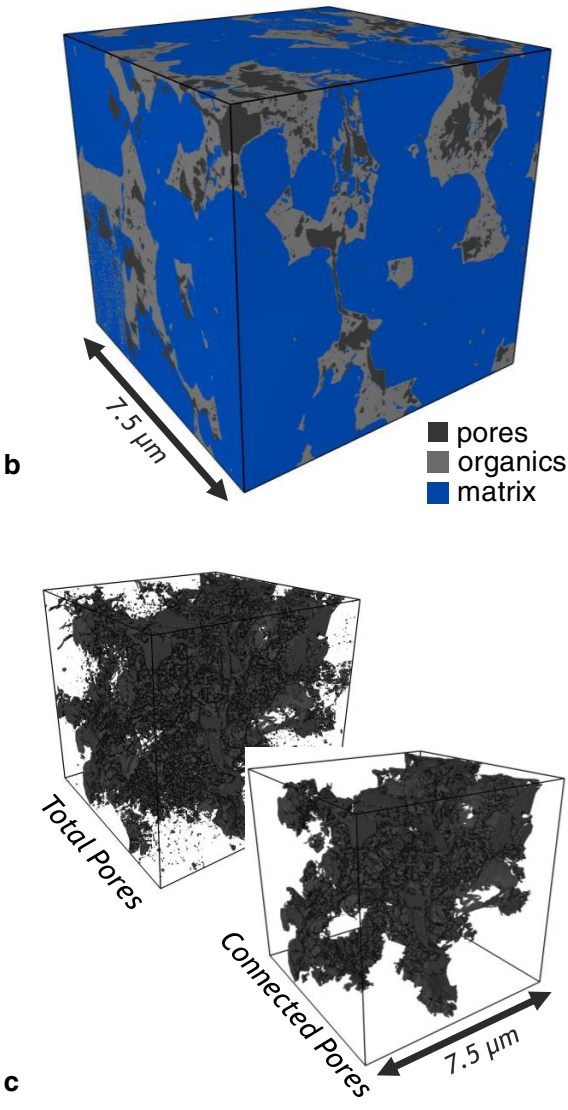

Figure 1. Representative SEM images of carbonate-rich source rocks. (a) Gray-scale SEM slice showing pores (black), organics (dark gray), matrix (light gray), and high-density minerals (white). The inset scale bar shows $5 \mu \mathrm{m}$. (b) Segmented 3D FIB-SEM volume showing pores (dark gray), organics (light gray), and matrix (blue). (c) Total pores and connected pores from the segmented $3 \mathrm{D}$ volume shown in (b). Note the labeled dimension on the volumes in $1 \mathrm{~b}$ and $1 \mathrm{c}$. 

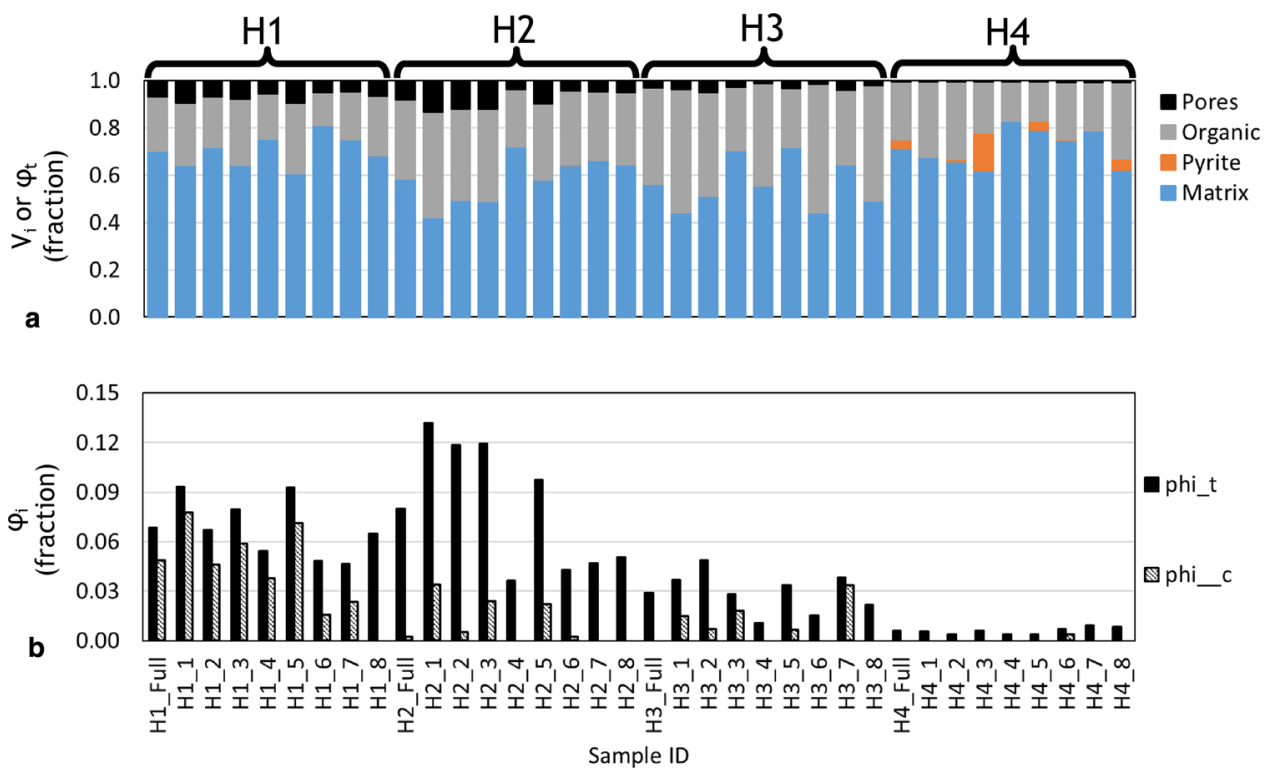

Figure 2. Composition of full and sub-sectioned from segmented 3D FIB-SEM rock volumes. (a) Volume fraction of total pores (i.e., pores), organic matter, pyrite, and matrix components. (b) Total porosity $\left(\phi_{t}\right.$, phi_t $)$ and connected porosity $\left(\phi_{c}\right.$, phi_c).

composition, total and connected porosity, and simulated data.

\section{Finite-volume method simulations}

Finite-volume method simulations of the effective thermal and electrical conductivity of the composite carbonate-rich source rock volumes in the X-, Y-, and Z-directions were performed using the ConductoDict module in GeoDict ${ }^{\circledR}$ (Math2Market). The thermal and electrical conductivity of the constituent materials and pore-filling high salinity water were assigned from bulk materials properties from the literature (Table S2). Thermal conductivity simulations solve Fourier's law with a temperature gradient of $2^{\circ} \mathrm{C}$ assuming pure diffusion and local thermal equilibrium, and electrical conductivity simulations solve Ohm's law with a potential difference of $1 \mathrm{~V}$. The effective thermal and electrical conductivities in all three directions are compared to the composition and porosity variations and used to assess microscale anisotropy.

\section{Empirical correlations and effective medium theory models}

Simulation results are fit with empirical correlations and compared to effective medium theory (EMT) models. For layered materials, the Voigt and Reuss bounds ${ }^{[25,26]}$ are used to define the upper and lower bounds of the effective thermal $(\lambda)$ and electrical $(\sigma)$ conductivity. ${ }^{[8]}$ The upper bound $\left(\lambda_{\|}\right.$or $\left.\sigma_{\|}\right)$of the effective conductivity can be modeled as a parallel layering as shown in Eq. 1 where $\lambda_{i}$ or $\sigma_{i}$ and $V_{i}$ are the conductivity and volume fraction of each component.

$$
\lambda_{\|}=\sum_{i} V_{i} \lambda_{i}
$$

$$
\sigma_{\|}=\sum_{i} V_{i} \sigma_{i}
$$

The lower bound $\left(\lambda_{\perp}\right)$ can be modeled as a perpendicular layering as shown in Eq. 2.

$$
\begin{aligned}
& \lambda_{\perp}=\left[\sum_{i} V_{i} \lambda_{i}^{-1}\right]^{-1}, \\
& \sigma_{\perp}=\left[\sum_{i} V_{i} \sigma_{i}^{-1}\right]^{-1} .
\end{aligned}
$$

The Krischer-Esdorn equation ${ }^{[27]}$ can be used to model the effective thermal conductivity between these two bounds as shown in Eq. 3 where $x$ is related to how much of the system resembles the perpendicular- or parallel-layered models ${ }^{[8]}$ (Eqs. 1a and 2a).

$$
\lambda_{K E}=\left[\frac{x}{\lambda_{\perp}}+\frac{1-x}{\lambda_{\|}}\right]^{-1} .
$$

The Lichtenecker and Rother equation ${ }^{[28]}$ can also be used to model the effective conductivity between the two bounds as shown for effective electrical conductivity in Eq. 4 where $\alpha$ similar to $x$ in Eq. 3 is related to rock texture. ${ }^{[8,29]}$

$$
\sigma_{L R}=\left[\sum_{i} V_{i} \sigma_{i}^{\alpha}\right]^{1 / \alpha} .
$$


Conventional reservoir rocks are modeled as two-component systems with the matrix minerals as the high thermal and zero electrical conductivity component and high salinity water as the low thermal and high electrical conductivity component.

Archie's equation $^{[30]}$ (Eq. 5), originally developed for clean sands, relates the conductivity of a fluid-saturated rock $\left(\sigma_{0}\right)$ to the conductivity of the fluid $\left(\sigma_{w}\right)$, porosity $\left(\phi_{t}\right)$, and complexity of the pore structure which is captured in the cementation exponent, $m$, and pre-factor, $a$.

$$
\frac{\sigma_{0}}{\sigma_{w}}=a \phi_{t}^{m}
$$

When Eq. 4 is applied to water saturated clean sands, $m=1 / \alpha$. In clean sands, the porosity $\left(\phi_{t}\right)$ is well connected and the high salinity fluid saturating the rock is the most conductive component and, thus, provides the conductive pathway.

In unconventional reservoir rocks, such as the carbonaterich mudstones sampled for this study, the connectivity of the pores can vary significantly. Thus, the electrical conductivity is related to the connected porosity $\left(\phi_{c}\right)$ rather than the total porosity $\left(\phi_{t}\right)$ as shown in Eq. 6. ${ }^{[29]}$

$$
\frac{\sigma_{0}}{\sigma_{w}}=a \phi_{c}^{m}
$$

\section{Results}

Figure 1 shows representative images of the samples used for this study. Figure 1(a) shows a single 2D SEM image with each component type identified. The gray-scale variations in the images are used to identify intensity ranges for image segmentation to digitally separate the pores, organics, highdensity minerals (i.e., pyrite), and matrix minerals (i.e., calcium carbonate). Figure 1(b) shows a representative segmented and labeled 3D image volume. The segmented 3D volumes and subvolumes were used as the input for the finite-volume method simulations. Finally, Fig. 1(c) shows the total and connected pores present in the volumes shown in Fig. 1(b). These images show that there is a significant difference between the total and connected porosity in these samples.

Each full rock volume has calcite with varying amounts of organic matter, pores, and pyrite which is the high-density mineral (Table S1). Figure 2(a) shows the composition of the full rock volumes and sub-volumes, all of which are referred to as samples moving forward. From the individual sample compositions, it can be seen that generating sub-volumes increases the number of samples and improves the heterogeneity of the sampled volumes. The $\mathrm{H} 1$ and $\mathrm{H} 4$ samples contained $20-40 \%$ organic matter, while the $\mathrm{H} 2$ and $\mathrm{H} 3$ samples contained considerably more organic matter, ranging from $30 \%$ to as high as $60 \%$ of the total rock volume. The $\mathrm{H} 2$ samples contain minor amounts of pyrite, while the $\mathrm{H} 4$ samples contained more pyrite, almost $20 \%$ in one case. Figure 2(b) compares the total porosity to connected porosity for all samples. All showed less than $15 \%$ total porosity and $8 \%$ or less connected porosity. About half of the samples did not contain connected porosity observable at this resolution.

The electrical and thermal conductivity of the components and the pore-filling fluids (Table S2) impact composite properties measured at any scale including those predicted by DRP simulation. The simulation results included in this study assume high salinity water as the pore-filling fluid; our previous work demonstrates the impact of changing fluid properties on the electrical conductivity. ${ }^{[20,23]}$ Figure 3(a) and (b) shows the simulation results for thermal and electrical conductivity in each direction, showing that both properties vary across the samples and with orientation. The thermal conductivity of the $\mathrm{H} 4$ samples is highest while that of the $\mathrm{H} 2$ and $\mathrm{H} 3$ samples tends to be lower. The H1 samples have the highest electrical conductivity while the $\mathrm{H} 3$ and $\mathrm{H} 4$ are the least conductive apart from few instances where the electrical conductivity in a single direction is similar to that of the $\mathrm{H} 1$ samples. These thermal and electrical conductivity variations are discussed next.

For porous metamorphic and sedimentary rocks, empirical relationships have been developed showing that the thermal conductivity decreases linearly with increasing porosity. ${ }^{[8]}$ Figure S2a shows the DRP-predicted thermal conductivity versus porosity for these carbonate-rich source rocks. For total porosity greater than $5 \%$, the thermal conductivity did decrease linearly with increasing porosity, but this trend did not hold for porosity less than 5\%. Figure S2a also shows that these data violate the upper and lower EMT bounds (Eqs. 1, 2) when approximating these samples as a calcite matrix containing pores filled with high salinity fluid as would be common for conventional reservoir rocks. In these samples, however, the porosity development is strongly correlated with organic content and the organic matter is the least conductive component. Figure S2b shows that, for these samples, the thermal conductivity linearly decreases with increasing organic matter content. Thus, the effective medium theory model can be applied by substituting organic matter content for the fluidfilled porosity. Figure $3 \mathrm{c}$ shows the thermal conductivity versus organic matter content with the EMT bounds and mixing relationships from Eqns. 1, 2, and 3. When represented this way, the EMT bounds are not violated and a simplified model can be developed using Eq. 3 to estimate the thermal conductivity from organic matter content. This shows that lower organic content tends toward lower values of the textural parameter, $x$, implying closer structural behavior to a parallel-layered model, while those with more organic matter where the parameter is closer to 0.5 implying mixed behavior. In all cases, the thermal conductivity in the Y- and Z-directions is similar, while the difference between these data and the values in the $\mathrm{X}$-direction increases with increasing organic content. One sample, at $21.4 \%$ organic content, does not follow the behavior and does violate the bounds. Upon further examination, this was found to be sample H4_3 which contains the highest pyrite fraction, $16.1 \%$, which has $4 \times$ higher thermal conductivity than calcite making the overall matrix thermal conductivity higher than that of the other samples. 

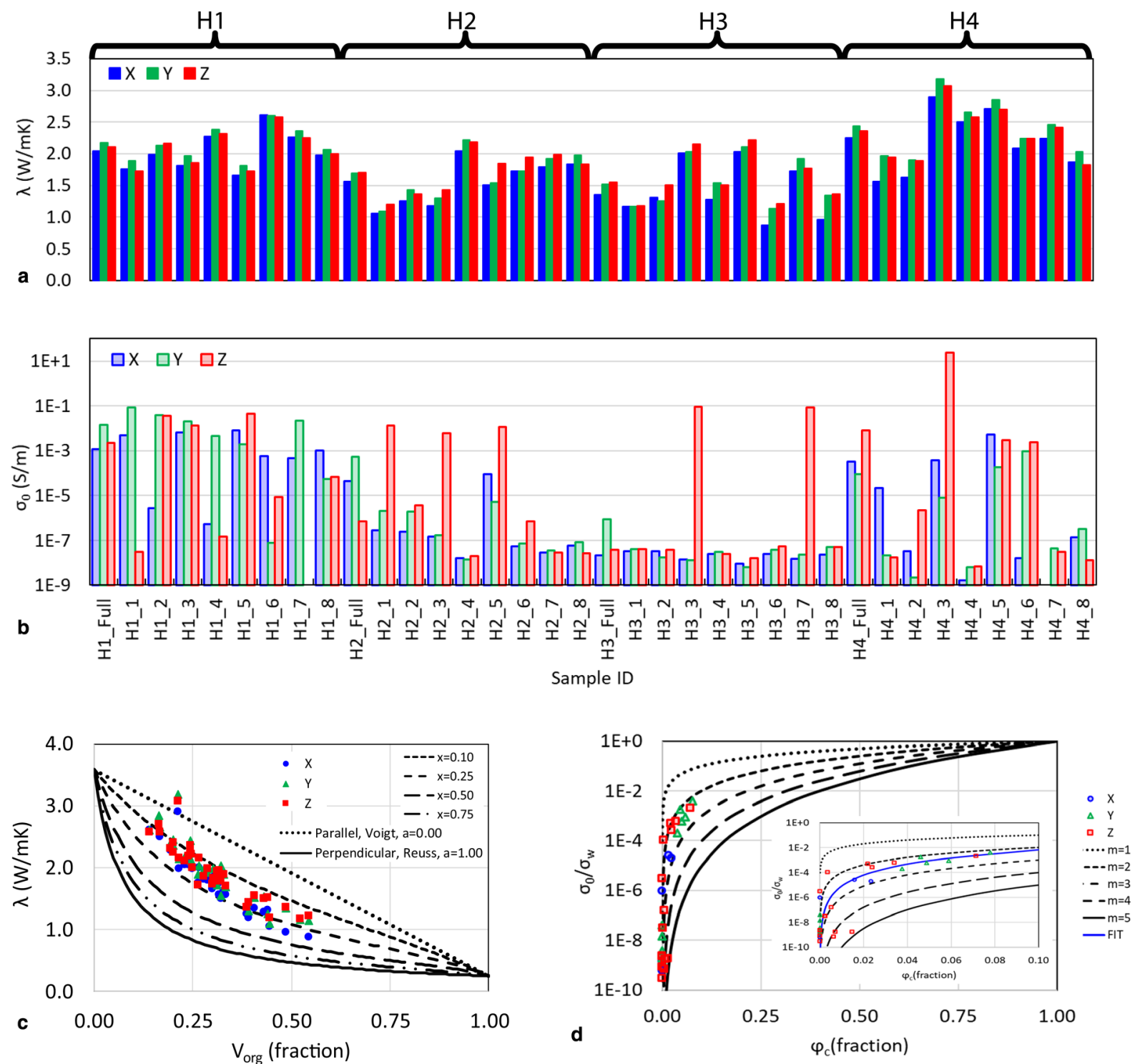

Figure 3. Results and property correlations. (a, b) DRP-predicted thermal and electrical conductivity in the X-, Y-, and Z-directions for each total and sub-sectioned rock volume. (c) Thermal conductivity versus organic matter content $\left(V_{\text {org }}\right)$ comparison showing data for all samples in each direction and EMT models from Eqs. 1-3. (d) Electrical conductivity versus connected porosity fraction in the direction of highest conductivity including Archie's model from Eq. 5 with $a=1$. Inset highlights the low connected porosity region and includes a fit to the data.

Archie's equation ${ }^{[30]}$ (Eq. 5) was developed for clean sands to describe the relationship between the electrical conductivity of the rock and the porosity in relation to the electrical conductivity of the pore-filling fluid. ${ }^{[8]}$ Figure S3a shows the DRP-predicted electrical conductivity versus total porosity in all three directions showing that an Archie-like dependence on total porosity is not observed. For clean sands, however, the total porosity is typically well connected and in the case of electrical conductivity, the high salinity water filling these connected pores provides the conductive pathway. Figure S3b shows the electrical conductivity in all three directions versus connected porosity. A trend is observed in the higher electrical conductivity data (i.e., $\sigma_{0}>1 \mathrm{E}-05$ ), but significant scatter is present in the samples with lower connected porosity. Note that comparable connectivity in all directions is not common in these samples. When considering that the connected porosity calculation is not direction specific, it is evident that these lower electrical conductivity data are related to constricted or non-existent connected porosity in the specified direction. In addition, some samples can be well connected in a single direction while others are well connected in two directions. Figure 3(d) shows the maximum electrical conductivity versus connected porosity for each sample. The solid lines show variations in the cementation exponent, $m$, with the pre-factor $a$ equal to 1 using Eq. 6 . The inset shows the data and a fit to Eq. 6 with a good correlation, $R^{2}=0.884$. The observed scatter in those data with the least and no connected porosity is related to the 
heterogeneity of the surrounding matrix as well as the structure of the connected pathway. For example, samples with limited connected porosity would contain varying amounts of organic matter and pyrite in addition to varying pore sizes and tortuosity. The values of pre-factor, $a$, and the cementation exponent, $m$, are typically related to the complexity of the pore structure. The prefactor, $a$, from these data is 5.7, while the cementation exponent, $m$, is 2.9 , both of which indicate complex pore structures. ${ }^{[8,29]}$

Structural anisotropy was not readily obvious in the $3 \mathrm{D}$ images of these samples due to the relatively small field of view. Given that the source rock samples used in this study are collected from a laminated system, anisotropy in the properties measured at the larger scales are ubiquitous. The anisotropy is calculated for each direction combination (i.e., XY, XZ, YZ) to test whether the effects of anisotropy on the measured properties are evident at this scale. Figure 4 shows the maximum anisotropy calculated in the DRP-predicted thermal and electrical conductivity for each sample. While the thermal conductivity of all samples shown in Fig. 3(a) is of similar scale in all directions, the results in Fig. 4(a) show some anisotropy is present even at this scale. The samples with the highest organic content (see Fig. 2(a), H3_6 and H3_8 for example) tend to have the highest anisotropy which would be expected given the depositional process and subsequent compaction that creates the reservoir environment. This can be seen in Fig. 3(c) and Fig. S2 where the samples with the highest organic content (i.e., $\left.V_{\text {org }}>0.3\right)$ tend to have similar thermal conductivity in the $\mathrm{Y}$ - and $\mathrm{Z}$-directions and lower thermal conductivity in the $\mathrm{X}$-direction. In addition, the $x$-value (Eq. 3 ) in the Y-and Z-directions tends toward lower values indicating parallel layering behavior while in the X-direction, the $x$-value (Eq. 3 ) tends toward 0.5 indicating mixed behavior. This process also impacts the connectivity of the pores at this scale since most of the porosity is developed in the organic matter during hydrocarbon generation. The electrical conductivity is correlated with the connected porosity when saturated with high salinity water. Figure 4(b) shows the maximum anisotropy of the electrical conductivity, where most samples show considerable anisotropy. Three of the four samples with the highest anisotropy H1_1, H3_3, and H3_7 have differing amounts of total and connected porosity but have high ratios of connected to total porosity. Interestingly, sample H4_3 has similarly high anisotropy but does not show connected pores at this resolution. This sample, however, has a large fraction of pyrite which constitutes a higher conductivity in the Z-direction (Fig. 4(b)). Samples with low connected porosity or significant constrictions along the connected pores tend to show low overall electrical conductivity due to the low conductivity of the organic and calcite components and lack of a continuous conductive pathway.

\section{Conclusion}

This work demonstrates the use of DRP to predict electrical and thermal conductivity of carbonate-rich source rocks from the Middle East using the same image volumes. While EMT and empirical correlations can be applied to these data, it is important to account for the appropriate materials and structural features. The relatively large amount of organic matter with low thermal conductivity controls the rock thermal conductivity as opposed to the fluid thermal conductivity as would be commonly assumed based on learnings from conventional rock systems. Similarly, the results show that Archie's equation can be applied to these samples by accounting for the direction and amount of connected porosity,
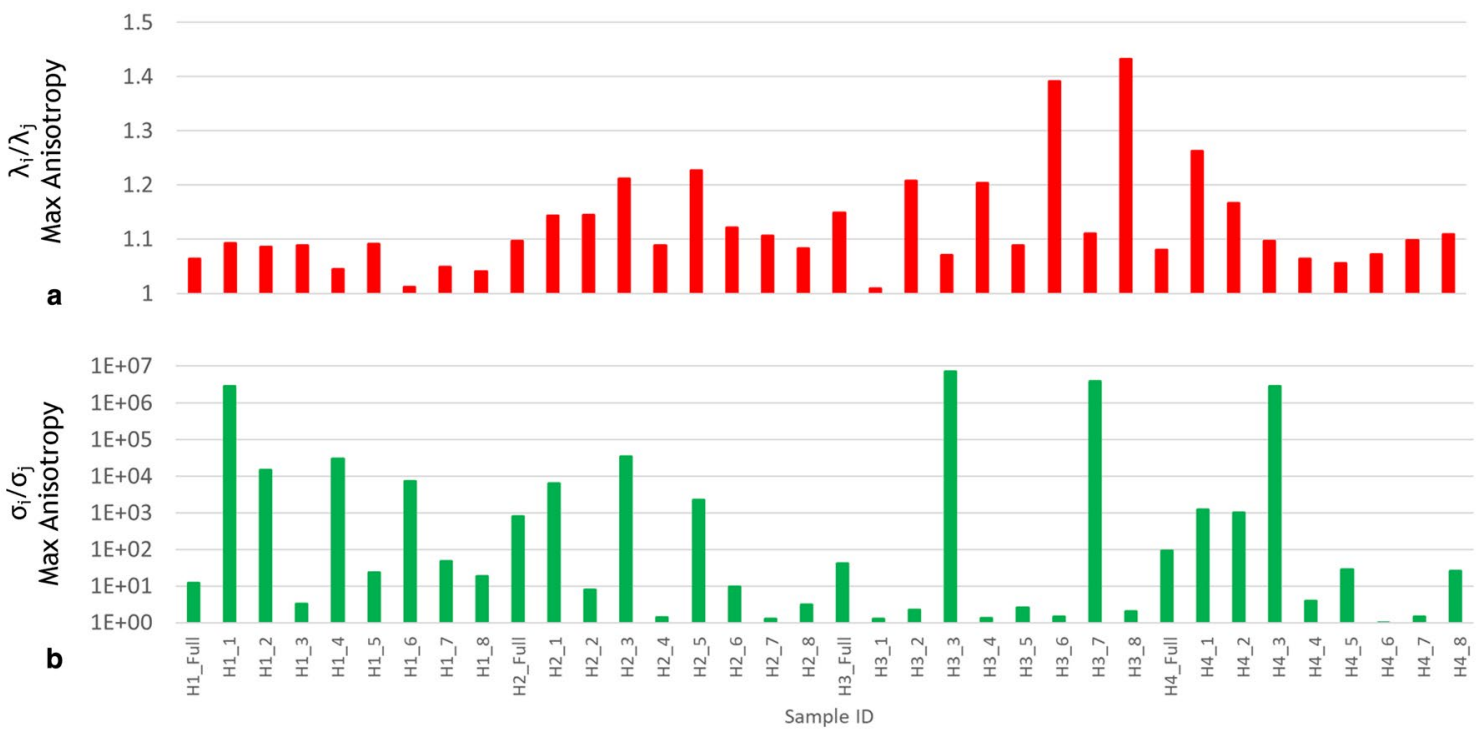

Figure 4. Maximum anisotropy for thermal conductivity (a) and electrical conductivity (b). 
especially when similar rock types are used. Results such as these help improve our understanding of how nanoscale heterogeneity can affect larger-scale measurements.

\section{Acknowledgments}

The authors would like to acknowledge support of Joshua Brothers and Jordan Kone for imaging in addition to the support of the Reservoir Engineering Technology Team at the Aramco Research Center-Houston for fruitful discussions.

\section{Data availability}

The datasets generated and/or analyzed during the current study are not publicly available due to company policy. In some circumstances, following company approval, some data may be made available from the corresponding author.

\section{Declarations}

\section{Conflict of interest}

The authors have no conflicts of interest to declare that are relevant to the content of this article.

\section{Open Access}

This article is licensed under a Creative Commons Attribution 4.0 International License, which permits use, sharing, adaptation, distribution and reproduction in any medium or format, as long as you give appropriate credit to the original author(s) and the source, provide a link to the Creative Commons licence, and indicate if changes were made. The images or other third party material in this article are included in the article's Creative Commons licence, unless indicated otherwise in a credit line to the material. If material is not included in the article's Creative Commons licence and your intended use is not permitted by statutory regulation or exceeds the permitted use, you will need to obtain permission directly from the copyright holder. To view a copy of this licence, visit http://creativecommons. org/licenses/by/4.0/.

\section{Supplementary Information}

The online version contains supplementary material available at https://doi.org/10.1557/s43579-021-00113-0.

\section{References}

1. L.T. Bryndzia, N.R. Braunsdorf, From source rock to reservoir: the evolution of self-sourced unconventional resource plays. Elements 10, 271 (2014)

2. K.E. Peters, M.R. Cassa, Applied Source Rock Geochemistry: Chapter 5: Part II Essential Elements (AAPG, Washington DC, 1994)

3. D.W. van Krevelen, Coal: Typology, Chemistry, Physics, Constitution (Elsevier, Amsterdam, 1961)

4. M. Vandenbroucke, Kerogen: from types to models of chemical structure. Oil Gas Sci. Technol. 58, 243 (2003)
5. T.J. Ahrens, Rock Physics \& Phase Relations: A Handbook of Physical Constants (American Geophysical Union, Washington, DC, 1995)

6. S. Fuchs, N. Balling, A. Förster, Calculation of thermal conductivity, thermal diffusivity and specific heat capacity of sedimentary rocks using petrophysical well logs. Geophys. J. Int. 203, 1977 (2015)

7. A. Hartmann, V. Rath, C. Clauser, Thermal conductivity from core and well log data. Int. J. Rock Mech. Min. Sci. 42, 1042 (2005)

8. J.H. Schön, Physical Properties of Rocks: Fundamentals and Principles of Petrophysics (Elsevier, Amsterdam, 2015)

9. L. Ma, A.-L. Fauchille, P.J. Dowey, F.F. Pilz, L. Courtois, K.G. Taylor, P.D. Lee, Correlative multi-scale imaging of shales: a review and future perspectives. Geol. Soc. Lond. 454, 175 (2017)

10. S. Kelly, H. El-Sobky, C. Torres-Verdín, M.T. Balhoff, Assessing the utility of FIB-SEM images for shale digital rock physics. Adv. Water Resour. 95, $302(2016)$

11. R.G. Loucks, R.M. Reed, S.C. Ruppel, D.M. Jarvie, Morphology, genesis, and distribution of nanometer-scale pores in siliceous mudstones of the Mississippian Barnett Shale. J. Sediment. Res. 79, 848 (2009)

12. K.L. Milliken, N.W. Hayman, Mudrock components and the genesis of bulk rock properties: review of current advances and challenges. Shale 2019, 1-25 (2019)

13. H. Andrä, N. Combaret, J. Dvorkin, E. Glatt, J. Han, M. Kabel, Y. Keehm, F. Krzikalla, M. Lee, C. Madonna, Digital rock physics benchmarks-Part II: computing effective properties. Comput. Geosci. 50, 33 (2013)

14. S. Berg, M. Rücker, H. Ott, A. Georgiadis, H. Van der Linde, F. Enzmann, M. Kersten, R. Armstrong, S. De With, J. Becker, Connected pathway relative permeability from pore-scale imaging of imbibition. Adv. Water Resour. 90, 24 (2016)

15. A.P. Byrnes, S. Zhang, L. Canter. M.D. Sonnenfeld, in Application of integrated core and multiscale 3-D image rock physics to characterize porosity, permeability, capillary pressure, and two- and three-phase relative permeability in the Codell Sandstone, Denver Basin, Colorado, in Unconventional Resources Technology Conference, Houston, Texas, 23-25 July 2018 (Society of Exploration Geophysicists, American Association of Petroleum Geologists, Society of Petroleum Engineers2018), pp. 3111.

16. Y. Hui, S. Lee, Y. Chen, M. Mahoney, J. Yu, Using three-dimensional image analysis techniques to understand the formation of the plastic layer during the heating of Australian coking coal blends. Energy Fuels 34, 3153 (2020)

17. A. Jacob, M. Peltz, S. Hale, F. Enzmann, O. Moravcova, L.N. Warr, G. Grathoff, P. Blum, M. Kersten, Simulating permeability reduction by clay mineral nanopores in a tight sandstone by combining computer $x$-ray microtomography and focussed ion beam scanning electron microscopy imaging. Solid Earth 12, 1 (2021)

18. N. Saxena, R. Hofmann, F.O. Alpak, S. Berg, J. Dietderich, U. Agarwal, K. Tandon, S. Hunter, J. Freeman, O.B. Wilson, References and benchmarks for pore-scale flow simulated using micro-CT images of porous media and digital rocks. Adv. Water Resour. 109, 211 (2017)

19. X. Zheng, X. Guo, Y. Yang, Z. Fu, K. Du, C. Wang, Y. Yi, Structure-dependent analysis of nanoporous metals: clues from mechanical, conduction, and flow properties. J. Phys. Chem. C 122, 16803 (2018)

20. A. Kotb, S.L. Eichmann, M. Sengupta, in Scale-dependent correlation to improve water saturation and permeability estimates in unconventional reservoirs, in Unconventional Resources Technology Conference, Denver, Colorado, 22-24 July 2019 (Unconventional Resources Technology Conference (URTeC); Society of Petroleum Engineers 2019), pp. 3001.

21. A. Mehmani, S. Kelly, C. Torres-Verdín, Leveraging digital rock physics workflows in unconventional petrophysics: a review of opportunities, challenges, and benchmarking, in SPWLA 60th Annual Logging Symposium (OnePetro2019).

22. V. Shabro, S. Kelly, C. Torres-Verdín, K. Sepehrnoori, A. Revil, Pore-scale modeling of electrical resistivity and permeability in FIB-SEM images of organic mudrock. Geophysics 79, D289 (2014)

23. S.L. Eichmann, A. Kotb, M. Sengupta, Digital rock evaluation of the thermal conductivity and electrical resistivity of source rocks. Microsc. Microanal. 25, 2424 (2019)

24. A. Hakami, A. Al-Mubarak, K. Al-Ramadan, C. Kurison, I. Leyva, Characterization of carbonate mudrocks of the Jurassic Tuwaiq Mountain 
Formation, Jafurah basin, Saudi Arabia: implications for unconventional reservoir potential evaluation. J. Nat. Gas Sci. Eng. 33, 1149 (2016)

25. A. Reuss, Berechnung der fließgrenze von mischkristallen auf grund der plastizitätsbedingung für einkristalle. ZAMM-J. Appl. Math. Mech./ Zeitschrift für Angewandte Mathematik und Mechanik 9, 49 (1929)

26. W. Voigt, Lehrbuch der kristallphysik: (mit ausschluss der kristalloptik), (BG Teubner1910).

27. O. Krischer, H. Esdorn, Die Wärmeübertragung in feuchten, porigen Stoffen verschiedener Struktur. Forschung auf dem Gebiet des Ingenieurwesens A 22, 1 (1956)
28. K.V. Lichtenecker, Die Herleitung des logarithmischen Mischungsgesetzes aus allgemeinen Prinzipien der stationaren Stromung. Phys. Z 32, 255 (1931)

29. G. Mavko, T. Mukerji, J. Dvorkin, The Rock Physics Handbook (Cambridge University Press, Cambridge, 2020)

30. G.E. Archie, The electrical resistivity log as an aid in determining some reservoir characteristics. Trans. AIME 146, 54 (1942) 